CLINICAL STUDY

\title{
Heterogeneity in responsiveness of perceived quality of life to body composition changes between adult- and childhood-onset Japanese hypopituitary adults with GH deficiency during GH replacement
}

\author{
Hisashi Urushihara ${ }^{1,2}$, Shunichi Fukuhara ${ }^{1}$, Shigeru Tai $^{2}$, Satoshi Morita ${ }^{1}$ and Kazuo Chihara ${ }^{3}$ \\ ${ }^{1}$ Department of Epidemiology and Healthcare Research, Kyoto University School of Public Health, Yoshida Konoe-cho, Sakyo-ku, Kyoto 606-8501, Japan, \\ ${ }^{2}$ Eli Lilly Japan K.K., Kobe 651-0086, Japan and ${ }^{3}$ Department of Clinical Molecular Medicine, Kobe University Graduate School of Medicine, Kobe \\ 650-0017, Japan \\ (Correspondence should be addressed to H Urushihara; Email: h.uru@st.pbh.med.kyoto-u.ac.jp)
}

\begin{abstract}
Objective: To examine the responsiveness of quality of life (QoL) associated with changes in clinical indices relevant to GH deficiency (GHD) in Japanese hypopituitary adults.

Design and methods: QoL was determined using the Short Form (SF)-36 in Japanese adults with adult(AO; $n=27$ ) or childhood- (CO; $n=37$ ) onset GHD in a 24-week double-blind placebo-controlled study with a fixed $\mathrm{GH}$ dose, and a subsequent 48-week open-label extension study with GH doses individualized using serum IGF-I levels.

Results: Baseline QoL was significantly decreased from the Japanese national reference in both onset types, more so in AO patients. Throughout the study, AO patients showed a trend for an increase in physical functioning and general health $(P=0.0564$ and 0.0999 respectively), whereas CO patients showed no changes in these domains. Fat mass changes negatively correlated with the changes in physical functioning and general health in AO patients $(r=-0.42$ and -0.64 respectively), but to a lesser degree in $\mathrm{CO}$ patients $(r=-0.36$ and -0.32 respectively). CO patients displayed significant decreases in social functioning $(P=0.0305)$ and mental health $(P=0.0442)$ and a decreasing trend in bodily pain $(P=0.0769)$, although no correlation between these decreases and any measured clinical index was observed, except between changes in bodily pain and IGF-I levels $(r=-0.43)$.

Conclusions: QoL impairment was evident in Japanese adults with GHD, particularly in AO patients. In AO patients, general health and physical functioning domains were responsive to fat mass changes during GH treatment; this association was not evident in CO patients. These relationships between QoL and body composition warrant verification.
\end{abstract}

European Journal of Endocrinology 156 637-645

\section{Introduction}

Growth hormone deficiency (GHD) in adult life causes various metabolic and psychological disturbances, resulting in an increased risk of cardiovascular morbidity/mortality and a reduced perception of quality of life (QoL; (1)). Affected QoL in adults with GHD has been generally noted in the areas of vitality, physical mobility, sleep, satisfaction with body shape, and sexual drive. GH replacement therapy for adult patients was registered in Western countries more than a decade ago and its effects on QoL have been extensively studied in Caucasian patients. Results have not always been consistent, however, possibly due to methodological differences such as in the optimal dosing of GH, sensitivity of the QoL instrument to the specific psychosocial symptoms of GH deficient adults, and differences in the populations studied (2-4). In particular, the time of onset of GHD has been shown to be an important factor that influences the outcome of GHD and GH replacement. Patients with childhoodonset (CO) GHD have less impaired QoL, despite apparent metabolic disturbance, somatic immaturity, and psychological and cognitive impairment, and have been shown to be less responsive to $\mathrm{GH}$ replacement with respect to QoL compared with adult-onset (AO) patients (5). In Japan, the adult indication of $\mathrm{GH}$ replacement became available as recently as April 2006, and thus only limited QoL data for Japanese patients are available (6).

In quantifying QoL in adult patients with GHD, generic questionnaires such as the Short Form (SF)-36 have been frequently criticized for a purported lack of sensitivity to the specific concerns to individuals with GHD; for this reason, the disease-specific questionnaires have been developed. Responsiveness, or sensitivity to 
change, is an important aspect of outcome measures such as QoL. There are two major aspects of responsiveness: one is 'internal responsiveness', the ability of the instrument to detect treatment-induced changes over time, and the other is 'external responsiveness', which reflects the extent to which changes in a measure over a specified time frame relate to corresponding changes in a reference measure of health status (7). When a certain change in the reference is accepted as indicating a change in the patient's condition, a corresponding change in the measure under investigation is regarded as clinically meaningful. A prerequisite for longitudinal assessment is to determine whether the changes in a QoL instrument accurately correspond to changes in a patient's health status.

In previous reports on Japanese hypopituitary adults with GHD (8, 9), it was demonstrated that the effects of $\mathrm{GH}$ replacement on body composition were comparable to those seen in Caucasian patients. QoL before and after $\mathrm{GH}$ replacement was assessed with the SF-36 in these studies, whose results have not been reported thus far. In the present paper, we report the responsiveness of QoL under GH replacement in a Japanese adult GHD population, with emphasis on whether the changes in clinical indices relevant to GHD were reflected in observed changes in scores of the eight SF-36 subscales.

\section{Patients and methods}

Two consecutive studies were conducted over 72 weeks, a 24-week randomized, placebo-controlled, doubleblind study with a fixed dose regimen, followed by a 48-week open-label study in which GH dose was individualized by serum insulin-like growth factor-I (IGF-I) level. A total of $27 \mathrm{AO}$ and $37 \mathrm{CO}$ hypopituitary patients with GHD from 25 investigative sites in Japan were enrolled. The studies were performed with appropriate local ethical approval according to the Declaration of Helsinki and all enrolled patients gave informed consent to participate in both the studies. Details of diagnosis, exclusion criteria, concomitant therapy including other hormone replacement, dose regimen, and clinical observations for both studies have been reported previously $(8,9)$.

The 64 Japanese patients ranged from 18 to 63 years. Eligibility criteria included diagnosis of a peak $\mathrm{GH}$ value $<3 \mu \mathrm{g}$ /l during a standard stimulation test such as with insulin or arginine. Major exclusion criteria were active malignancy, diabetes mellitus, and uncontrolled hypertension. Adequate replacement for pituitary hormonal deficiencies other than $\mathrm{GH}$ was ensured for at least 3 months before enrolment and throughout both the studies. During the double-blind phase, 14 AO and 19 CO patients were treated for 24 weeks with a fixed dose regimen of recombinant human $\mathrm{GH}$ (Humatrope, Eli Lilly and Company), given as six or seven injections per week. Doses were started at $0.021 \mathrm{mg} / \mathrm{kg}$ per week, subsequently escalated to 0.042 and finally $0.084 \mathrm{mg} / \mathrm{kg}$ per week. The remaining $13 \mathrm{AO}$ and 18 CO patients received equivalent volumes of placebo. Two AO patients in the GH course and two AO and one CO patient in the placebo course, discontinued treatment prior to completion of the double-blind study. During the open-label extension phase, 59 patients received $\mathrm{GH}$, at final individualized doses ranging from 0.021 to $0.084 \mathrm{mg} / \mathrm{kg}$ per week (9), with $23 \mathrm{AO}$ and 33 CO patients completing the extension study and three CO patients discontinuing before completion.

To measure perceived QoL, the Japanese SF-36v2 Health Survey (10) was administered at baseline, at completion of the double-blind study, and at completion of the open study. To obviate physician influence, the patient completed the questionnaire and returned it in a sealed envelope to hospital staff before interview with the attending physician. Body composition was determined at 24-week intervals throughout the studies by dual-energy X-ray absorptiometry (DXA), as previously described $(8,9)$. Blood sampling for measurement of serum IGF-I levels and other laboratory parameters was also performed at the scheduled visits, with the assays performed centrally $(8,9)$.

In the double-blind phase, all $37 \mathrm{CO}$ patients from both treatment courses completed the questionnaires. Among AO patients, 13 of 14 patients in the GH group $(92.9 \%)$ and 12 of 13 in the placebo group (92.3\%) returned the questionnaires; 1 patient in the GH group was unable to fill in the questionnaire at discontinuation due to cerebral infarction, while a second in the placebo group was excluded from analysis due to protocol violation. Response rate to the questionnaire for the $23 \mathrm{AO}$ and $36 \mathrm{CO}$ patients in the open-label phase was $100 \%$.

Two scoring methods were utilized to analyze SF-36 subscales, with higher scores indicating better health status in both. First, the sum of item scores for each SF36 domain was transformed to a 0 (lowest value) to 100 (highest value) numerical scale (transformed scale). Second, these transformed SF-36 values were used to calculate norm-based scores (NBS), standardized for age- and gender-matched Japanese controls to a numerical mean of 50 and S.D. of 10 (10). Baseline QoL data are presented as NBS, which enables between subscale comparison of the degree of deviation from the norm. Changes during studies for the eight SF-36 dimensions are presented as changes in the 0-100 transformed scale. For responsiveness analysis, the patients were pooled regardless of the treatment course in the double-blind study and changes in SF-36 dimensions were calculated using transformed values as the difference between the baseline value at the start of the double-blind study and the last value observed in the open-label phase. 
Summary statistics are presented as mean \pm s.D. unless otherwise specified, with 95\% confidence intervals of the difference in means where appropriate. Baseline QoL scores were compared with the Japanese national mean of 50 using Student's $t$-test. Pairwise comparisons, such as differences in QoL values, body composition, and other clinical parameters between two time points, were performed by the paired $t$-test. Correlations with QoL scores were evaluated with Pearson's $r$ coefficient and presented if the absolute $r$ coefficient exceeded 0.4, which indicates moderate correlation. Standardized response mean (SRM), defined as mean change divided by the S.D. of the change, was used for evaluation of internal responsiveness of the subscales (7). To assess external responsiveness, the relationship of changes in clinical variables of interest with changes in SF-36 domains was examined by univariate regression analysis (7). Multivariate analysis examined changes in SF-36 subscales relative to changes in the external standard variable and baseline parameters such as gender, age, onset, treatment course, and baseline score. This model for external responsiveness also included the by-onset interaction. All results were assessed at a two-tailed significance level of 5\%, except for interaction, which was assessed at $15 \%$. Statistical analysis was performed with SPSS for Windows (Ver. 13.0, SPSS Inc., Chicago, IL, USA).

\section{Results}

\section{Baseline data}

Baseline characteristics of the 64 enrolled patients are summarized by GHD onset in Table 1. Differences by onset were observed in age, age at diagnosis, and serum
IGF-I level and its SD score. As shown in Fig. 1, AO patients scored significantly lower than normal in five of the eight SF-36 domains, i.e., from the lowest, general health $(P<0.001)$, role physical $(P=0.006)$, role emotional $(P=0.019)$, vitality $(P=0.008)$, and physical functioning $(P=0.046)$; in contrast, $\mathrm{CO}$ patients had significantly lower scores than the normal only in role physical as the lower and general health $(P=0.049$ and 0.041 respectively).

When the correlations between baseline transformed individual SF-36 scores and the clinical variables listed in Table 1 were examined, a moderate positive correlation was found in AO patients between the score for general health and peak value in the GH stimulation test $(r=0.43)$. In CO patients, physical functioning was found to be moderately correlated with fat mass, body mass index, and IGF-I SD score $(r=-0.47,-0.45$ and 0.42 respectively). In addition, social functioning was positively correlated with serum IGF-I SD score at baseline in CO patients $(r=0.45)$. Age was not correlated with baseline values of any of the eight domains in either $\mathrm{AO}$ or $\mathrm{CO}$ patients.

\section{Double-blind phase}

In $\mathrm{AO}$ patients, no differences in changes between $\mathrm{GH}$ and placebo-treated patients were seen in any of the SF36 domains. In CO patients, a significant difference between GH and placebo treatment was seen for the mental health subscale only $(P=0.045)$; however, the within-group change in the GH group was not significant (GH 2.6 $\pm 12.4, P=0.367$; placebo $-7.8 \pm$ 17.7, $P=0.079)$. In the GH-treated AO patients, a significant reduction in the bodily pain subscale was

Table 1 Baseline characteristics of Japanese adult hypopituitary patients with adult- (AO) and childhood- (CO) onset growth hormone (GH) deficiency.

\begin{tabular}{|c|c|c|}
\hline & AO $(n=27)$ & $\operatorname{Co}(n=37)$ \\
\hline Age (years) & $50.8 \pm 9.7$ & $28.8 \pm 7.3$ \\
\hline Age at diagnosis (years) & $38.5 \pm 11.3$ & $11.4 \pm 6.4$ \\
\hline Gender (male/female) & $9(33.3 \%) / 18(66.7 \%)$ & $22(59.5 \%) / 15(40.5 \%)$ \\
\hline Pituitary deficiency (isolated/multiple) ${ }^{\mathrm{a}}$ & $1 / 26$ & $0 / 37$ \\
\hline \multicolumn{3}{|l|}{ Etiology } \\
\hline Idiopathic & - & $15(40.5 \%)$ \\
\hline Tumor ${ }^{b}$ & $22(81.5 \%)$ & $20(54.1 \%)$ \\
\hline Sheehan syndrome & $4(14.8 \%)$ & - \\
\hline Empty sella & $1(3.7 \%)$ & $1(2.7 \%)$ \\
\hline Trauma & - & $1(2.7 \%)$ \\
\hline Height (mean, range; cm) & $160.5(144.0-180.7)$ & $163.9(147.4-180.4)$ \\
\hline Weight (kg) & $62.2 \pm 14.3$ & $65.0 \pm 16.0$ \\
\hline Body mass index $\left(\mathrm{kg} / \mathrm{m}^{2}\right)$ & $23.9 \pm 3.7$ & $24.1 \pm 4.9$ \\
\hline Lean body mass $(\mathrm{kg})$ & $38.2+9.1$ & $40.9+10.4$ \\
\hline Fat mass $(\mathrm{kg})$ & $21.2 \pm 6.6$ & $21.1 \pm 7.9$ \\
\hline Peak GH (mean, range; $\mathrm{ng} / \mathrm{ml})^{\mathrm{c}}$ & $0.5(0.1-2.7)$ & $0.3(0.1-1.4)$ \\
\hline IGF-I (ng/ml) & $92 \pm 50$ & $53 \pm 37$ \\
\hline IGF-I SD score & $-0.93 \pm 1.05$ & $-3.18 \pm 1.48$ \\
\hline
\end{tabular}

Values are expressed as either mean \pm s.D. or mean with range in parentheses.

asolated GHD or multiple pituitary deficiencies.

'Includes pituitary adenoma, craniopharyngioma, glioma, and germ cell cancer.

In standard stimulation tests. 


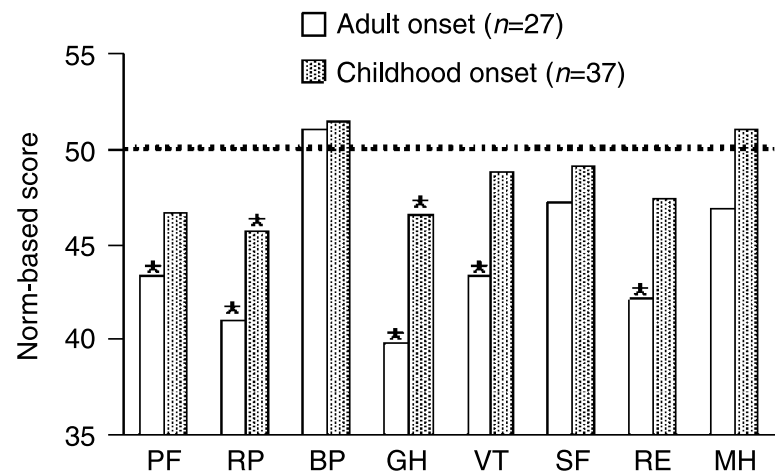

Figure 1 Baseline values of health-related QoL measured with the SF-36 by disease onset in Japanese adult hypopituitary patients with GH deficiency. Higher score indicates better perceived health; normbased score (NBS) is the S.D. score adjusted for age and gender having a mean and S.D. of 50 and 10 respectively. ${ }^{\star} P<0.05$ for comparison of mean NBS in either AO or CO patients at baseline with the Japanese national reference value of 50 . PF, physical functioning; $\mathrm{RP}$, role physical; BP, bodily pain; GH, general health; VT, vitality; SF, social functioning; $\mathrm{RE}$, role emotional; $\mathrm{MH}$, mental health.

seen from baseline to the end of the double-blind study (change $-12.8 \pm 20.6, P=0.044$ ), without a significant difference with the placebo group $(P=0.144)$.

\section{Change throughout the studies}

Change scores in the SF-36 subscales throughout the two successive studies are presented in Table 2. In AO patients, the change from baseline to endpoint was not significant in any of the eight QoL domains, although increasing trends were observed in physical functioning and general health $(P=0.0564$ and 0.0999 respectively). On the other hand, significant negative changes in social functioning $(P=0.0305)$ and mental health $(P=0.0442)$ were seen in CO patients, along with a trend to a decrease in bodily pain $(P=0.0769)$.

Mean doses were stable from 24 weeks after the initiation of individualized dose titration, and were finally maintained at the end of the open-label study at $0.034 \pm$ $0.022 \mathrm{mg} / \mathrm{kg}$ per week for AO patients and $0.060 \pm$
$0.021 \mathrm{mg} / \mathrm{kg}$ per week for $\mathrm{CO}$ patients. In parallel, mean serum IGF-I SD scores increased up to within the normal range in both onset groups (at the end of the open study: AO $1.17 \pm 1.22$, CO $-0.22 \pm 1.89$; change throughout the studies: AO 2.11 \pm 1.18, CO 2.99 $\pm 1.56, P<0.001$ for both). During GH replacement there was a significant reduction in fat mass in both onsets $(\mathrm{AO}-1.4 \pm 2.6 \mathrm{~kg}$, $P=0.018 ; C O-1.8 \pm 2.3 \mathrm{~kg}, P<0.001)$, with a significant increase in lean body mass (AO $1.3 \pm 1.9 \mathrm{~kg}$, $P=0.003$; CO $2.2 \pm 2.2 \mathrm{~kg}, P<0.001$ ). In AO patients, moderate correlations of net fat mass change with changes in scores for general health $(r=-0.64)$ and physical functioning ( $r=-0.42$ ) were found (Fig. 2), with similar but reduced correlations observed in CO patients (physical functioning: $r=-0.36$, general health: $r=-0.32$ ). No moderate correlations were found between changes in lean body mass and changes in the eight SF-36 subscales. In CO patients, changes in serum IGF-I SD score correlated with changes in bodily pain $(r=-0.43)$.

\section{Responsiveness}

SRM was calculated to evaluate internal responsiveness of the eight QoL domains in the SF-36. External responsiveness of these subscales was assessed with linear regression models using fat mass change as an independent variable. In AO patients, SRMs for physical functioning and general health subscales were greater than in other domains of the SF-36, and the SRM for physical functioning was close to 0.5 , indicating moderate internal responsiveness. In univariate regression analysis, the fat mass change in AO patients accounted for a significant amount of variance, at up to $41.2 \%$ for general health changes (Table 3). Other domains were not significantly responsive to change in fat mass. In CO patients, SRMs for physical functioning and general health were small, whereas external responsiveness to changes in fat mass was significant for physical functioning and marginal for general health. In contrast, bodily pain, social functioning,

Table 2 Quality of life (QoL) score changes between baseline and last observed value after growth hormone (GH) replacement measured with the Short Form (SF)-36 in Japanese hypopituitary adults with GH deficiency.

\begin{tabular}{|c|c|c|c|c|c|c|}
\hline \multirow[b]{2}{*}{ Subscale } & \multicolumn{3}{|c|}{ AO $(n=27)$} & \multicolumn{3}{|c|}{$\operatorname{CO}(n=37)$} \\
\hline & Baseline & $\begin{array}{l}\text { Mean change } \\
\quad(95 \% \mathrm{Cl})\end{array}$ & $P$ value $^{\mathrm{a}}$ & Baseline & $\begin{array}{l}\text { Mean change } \\
\quad(95 \% \mathrm{Cl})\end{array}$ & $P$ value ${ }^{\mathrm{a}}$ \\
\hline Physical functioning & $79.8 \pm 17.7$ & $4.5(-0.1,9.2)$ & 0.0564 & $90.8 \pm 12.3$ & $0.3(-1.8,2.3)$ & 0.786 \\
\hline Role physical & $72.9 \pm 29.0$ & $1.6(-7.8,11.1)$ & 0.725 & $87.7 \pm 16.5$ & $0.2(-4.7,5.0)$ & 0.943 \\
\hline Bodily pain & $75.6 \pm 24.5$ & $-2.7(-13.5,8.0)$ & 0.604 & $80.7 \pm 20.7$ & $-5.6(-11.8,0.6)$ & 0.0769 \\
\hline General health & $45.1 \pm 19.6$ & $7.7(-1.6,17.0)$ & 0.0999 & $61.3 \pm 18.1$ & $-0.8(-5.6,3.9)$ & 0.726 \\
\hline Vitality & $48.7 \pm 24.5$ & $3.8(-3.7,11.3)$ & 0.306 & $58.3 \pm 18.8$ & $-1.1(-5.5,3.3)$ & 0.616 \\
\hline Social functioning & $80.6 \pm 20.3$ & $-6.0(-16.1,4.2)$ & 0.235 & $85.5 \pm 21.1$ & $-7.6(-14.5,-0.8)$ & 0.0305 \\
\hline Role emotional & $72.4 \pm 31.1$ & $2.9(-3.9,9.7)$ & 0.389 & $84.9 \pm 17.0$ & $-0.5(-5.7,4.8)$ & 0.859 \\
\hline Mental health & $65.8 \pm 20.4$ & $0.0(-10.5,10.5)$ & 1.00 & $72.6 \pm 13.5$ & $-4.9(-9.7,-0.1)$ & 0.0442 \\
\hline
\end{tabular}

Baseline values are expressed as mean \pm S.D.

a Student's $t$-test; $P$ values are for significance of mean changes within group. Bold indicates $P<0.05$. 

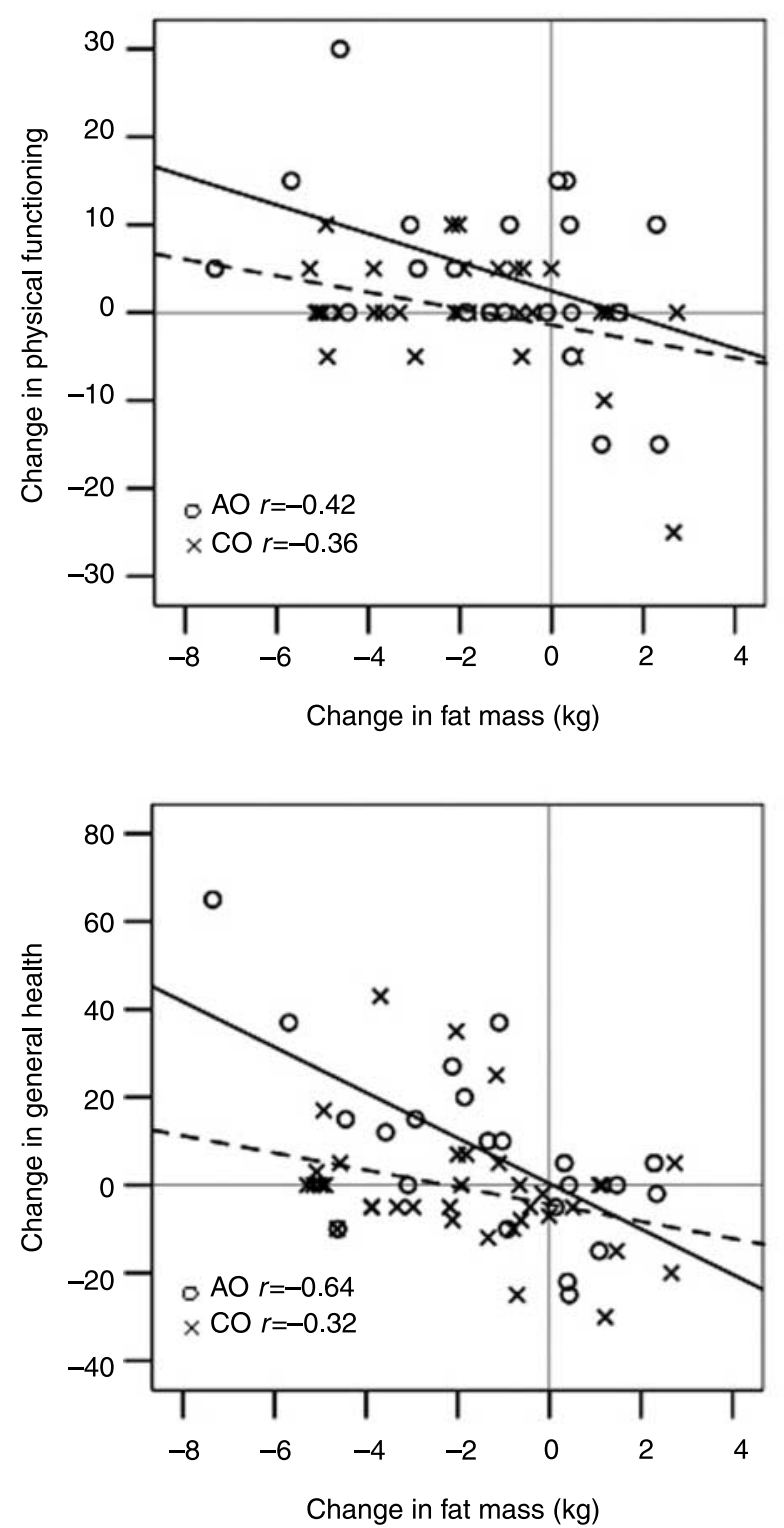

Figure 2 Correlation between changes in QoL score and changes in fat mass in $\mathrm{AO}$ and $\mathrm{CO}$ Japanese hypopituitary adults with $\mathrm{GH}$ deficiency. Superimposed lines show regression for $A O$ (solid) and CO patients (broken).

and mental health subscales showed greater SRMs in $\mathrm{CO}$ subjects but failed to be significantly responsive to fat mass change (Table 3). Lean body mass was not an adequate clinical index of external responsiveness of QoL domains as its changes failed to correlate with any of the subscale changes in either onset (data not shown). Change in serum IGF-I SD score throughout the studies was correlated with the change in bodily pain domain, and accounted for a significant amount of variance in the changes in bodily pain $\left(R^{2}=0.187, P=\right.$ $0.008)$ in $\mathrm{CO}$, but not in AO patients $(P=0.962)$.

In the univariate regression model for general health subscale to fat mass changes, the regression coefficient
(B) and intercept in the AO population were -5.17 and 0.37 respectively (Table 3, Fig. 2). From this model, a $2 \mathrm{~kg}$ net reduction in fat mass corresponded to a positive change in score of 10.7, and no change in fat mass resulted in almost no change in general health subscale. For CO patients, in contrast, a comparable change in the general health subscale required a reduction in fat mass of more than $7 \mathrm{~kg}$, and the change would be negative with no change in fat mass (B: -1.95 , intercept: -4.38 ), indicating heterogeneity between the two GHD entities in responsiveness of the perceived QoL to fat mass status.

To statistically investigate whether the effects of fat mass reduction on QoL change varied by onset, the by-onset interaction was examined with the multivariate models of physical functioning and general health using fat mass change as an independent variable (Table 4). Interaction by onset was insignificant $(P=0.345)$ in the model of physical functioning, which included gender, age, treatment course, baseline score, and baseline fat mass as covariates. The by-onset interaction with the effect of fat mass change on general health was significant at a level of 0.15 after adjustment for the covariates $(P=0.102)$. The effects of age or treatment course were not significant in either model.

\section{Discussion}

We studied QoL and its association with relevant clinical indices of GHD in adult Japanese patients using the Japanese SF-36 v2 Health Survey, a validated generic health-related QoL questionnaire for the Japanese. Results showed that the degree of QoL impairment at baseline in Japanese hypopituitary adults with GHD varied by onset, similar to Caucasian patients, and that positive changes in physical functioning and general health domains were significantly correlated with fat mass reduction under $\mathrm{GH}$ replacement.

The SF-36 questionnaire contains eight healthrelated domains, which allows the specific patterns of QoL deficit to be identified when a particular disease patient group is compared with a national normative reference. Our baseline data indicated that healthrelated QoL was affected in Japanese adult GHD patients, with apparent differences in the degree of QoL impairment between onsets after adjustment for age differences of onset. This finding is consistent with previous reports that QoL is more strongly affected in AO than CO patients $(5,11)$. In addition, our observation of impaired general health and physical functioning at baseline is consistent with previous findings in several cross-sectional studies using the SF36 in Caucasian hypopituitary patients $(12,13)$.

The QoL impairment observed in our Japanese CO patients was mild, at least in part due to insufficient sensitivity of the SF-36, a generic QoL questionnaire. In Caucasian patients with CO GHD, inconsistencies have 
Table 3 Internal and external responsiveness of eight subscales of the Short Form (SF)-36 under growth hormone (GH) replacement in Japanese hypopituitary adults with GH deficiency.

\begin{tabular}{|c|c|c|c|c|c|}
\hline \multirow[b]{3}{*}{ Onset } & \multirow[b]{3}{*}{ Subscale } & \multirow{3}{*}{$\begin{array}{l}\text { Internal responsiveness } \\
\qquad \mathrm{SRM}^{\mathrm{b}}\end{array}$} & \multicolumn{3}{|c|}{ External responsiveness $^{\mathrm{a}}$} \\
\hline & & & \multicolumn{3}{|c|}{ Linear regression } \\
\hline & & & $R^{2}$ & $B$ & $P$ value \\
\hline \multirow[t]{8}{*}{$\mathrm{AO}$} & Physical functioning & 0.442 & 0.176 & -1.63 & 0.059 \\
\hline & Role physical & 0.074 & 0.017 & -1.12 & 0.551 \\
\hline & Bodily pain & -0.110 & 0.001 & -0.25 & 0.909 \\
\hline & General health & 0.367 & 0.412 & -5.17 & 0.001 \\
\hline & Vitality & 0.224 & 0.049 & -1.45 & 0.321 \\
\hline & Social functioning & -0.254 & 0.011 & 0.99 & 0.626 \\
\hline & Role emotional & 0.183 & 0.045 & -1.31 & 0.334 \\
\hline & Mental health & 0.000 & 0.006 & -0.72 & 0.729 \\
\hline \multirow[t]{8}{*}{$\mathrm{CO}$} & Physical functioning & 0.046 & 0.129 & -0.93 & 0.031 \\
\hline & Role physical & 0.012 & 0.019 & 0.85 & 0.420 \\
\hline & Bodily pain & -0.304 & 0.020 & 1.11 & 0.410 \\
\hline & General health & -0.059 & 0.104 & -1.95 & 0.055 \\
\hline & Vitality & -0.084 & 0.048 & -1.22 & 0.199 \\
\hline & Social functioning & -0.376 & 0.030 & -1.49 & 0.316 \\
\hline & Role emotional & -0.030 & 0.004 & -0.40 & 0.727 \\
\hline & Mental health & -0.348 & 0.071 & -1.61 & 0.116 \\
\hline
\end{tabular}

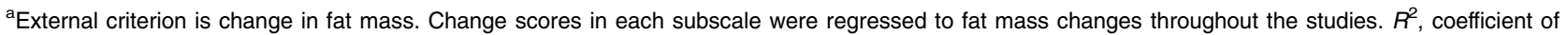
determination of model; $\mathrm{B}$, regression coefficient; $P$ values are for significance of regression coefficient. Bold indicates $P<0.05$.

${ }^{b}$ Standardized response mean (SRM) is defined as a mean change divided by the S.D. of the change.

been noted between perceived QoL impairment and adverse clinical and laboratory findings. Despite various psychological and cognitive deficits, as well as an abnormal lipid profile and central adiposity, young patients with $\mathrm{CO}$ GHD who discontinue pediatric GH replacement exhibit only mild impairment of QoL when compared with GH-sufficient peers $(14,15)$. Our CO patients have also consistently shown findings adverse to those characteristic of adult GHD, such as abnormal body composition and lipid profile at baseline (8). These findings may suggest another possibility, namely $\mathrm{CO}$ patients have adapted to the chronic effects of GHD during development and do not perceive their $\mathrm{QoL}$ as impaired $(5,14,16)$

Responsiveness, defined as the ability to detect a change due to a treatment, is an important characteristic of a QoL instrument (17). In particular, external responsiveness assesses the extent to which a change in a QoL instrument relates to clinically significant changes in known external standard health measures. Two subscales of the SF-36 were significantly responsive to changes in fat mass, whereas no subscale was

Table 4 Multivariate regression models of changes in physical functioning and general health subscale scores of Short Form (SF)-36 including interaction between onset and fat mass change.

\begin{tabular}{|c|c|c|c|}
\hline Change score & Variable & Parameter estimate & $P$ value \\
\hline \multirow[t]{8}{*}{ Physical functioning $\left(R^{2}=0.308\right)$} & Onset $(0-A O, 1-C O)$ & -0.91 & 0.828 \\
\hline & Gender (0-female, 1-male) & 0.75 & 0.715 \\
\hline & Age & 0.07 & 0.622 \\
\hline & Treatment group $^{\mathrm{a}}$ (0-hGH, 1-placebo) & -1.52 & 0.470 \\
\hline & Physical functioning at baseline & -0.15 & 0.033 \\
\hline & Fat mass at baseline $(\mathrm{kg})$ & -0.28 & 0.109 \\
\hline & Change in fat mass $(\mathrm{kg})$ & -1.57 & 0.026 \\
\hline & Interaction (onset, fat mass change) & 0.85 & 0.345 \\
\hline \multirow[t]{8}{*}{ General health $\left(R^{2}=0.390\right)$} & Onset $(0-A O, 1-C O)$ & -3.71 & 0.687 \\
\hline & Gender (0-female, 1-male) & -4.53 & 0.308 \\
\hline & Age & -0.67 & 0.835 \\
\hline & Age at diagnosis & -0.14 & 0.647 \\
\hline & Treatment group $^{\mathrm{a}}$ (0-hGH, 1-placebo) & 0.50 & 0.901 \\
\hline & General health at baseline & -0.17 & 0.120 \\
\hline & Change in fat mass $(\mathrm{kg})$ & -4.56 & 0.001 \\
\hline & Interaction (onset, fat mass change) & 2.97 & 0.102 \\
\hline
\end{tabular}

Onset, gender, age, treatment group, and the baseline value were incorporated in the model; clinical variables with correlation coefficient $r$ with change scores above 0.4 were also included in the models. $R^{2}$, coefficient of determination of the model; $P$ values are for significance of parameter estimates. Bold indicates $P<0.05$ for significance of parameter. Italics indicate $P<0.15$ for significance of interaction.

${ }^{a}$ Treatment group indicates drug allocation during the first double-blind study. 
responsive to those in lean body mass. Fat mass is one of the most responsive external markers of the effects of $\mathrm{GH}$ in patients with GHD. Nevertheless, no relationship between changes in QoL and changes in fat mass, lean body mass, or IGF-I has been found in a variety of studies $(11,18,19)$, except one which reported a significant correlation between body composition change as determined by bioelectrical impedance analysis and change in QoL score as measured with the disease-specific questionnaire, Quality of LifeAssessment of GHD in Adults (QoL-AGHDA) (20). Here, using DXA, we found significant associations between changes in general health and physical functioning subscales and fat mass change, with the trend being more consistent in AO than $\mathrm{CO}$ patients. Specifically, the by-onset interaction was significant in the model relating changes in general health to changes in fat mass, indicating a possible onset effect on external responsiveness to fat mass status. This differential responsiveness by onset type may be influenced by differences between $\mathrm{AO}$ and $\mathrm{CO}$ patients in the degree of baseline impairment in these domains. QoL improvements under GH replacement have in fact been reported to be more pronounced in patients with more severe impairment at baseline (21). In our multivariate model, although the change score for physical functioning was greater in patients with lower baseline scores, this was not the case for general health, for which baseline status did not influence changes despite considerable baseline differences between onsets. This finding suggests essential heterogeneity in responsiveness due to onset.

Increased fat mass, abdominal obesity, and poor body image are clinical features of GHD. Some studies have reported a significant association between baseline QoL score and body mass index $(22,23)$. In semi-structured interviews, adult patients with GHD report excess fat mass as a major reason for poor body image and thus body image has been included as a major item in GHDspecific questionnaires, such as AGHDA (24) and Questions on Life Satisfaction-Hypopituitarism (QLS-H) (25). The strong association of the general health subscale with fat mass status in our studies is consistent with these findings, although the possibility of chance correlation cannot be excluded. A similar relationship between obesity and QoL has been sought in several clinical contexts, including: a cross-sectional study in a normal Dutch population, which found associations between higher body mass index and lower general health in women and between larger weight circumference and lower physical functioning of the SF-36 (26); a study in healthy Dutch postmenopausal women which reported that fat mass was negatively associated with QLS-health subscale whereas lean body mass was not (27); and studies on the effects of surgery for severe obesity (28).

Significant negative changes were seen in social functioning and mental health subscales in CO patients without external responsiveness to changes in any
GH-related parameter. However, it is possible that the expected positive effects of GH replacement on psychosocial functioning $(29,30)$ could not be detected due to changes in QoL unrelated to GHD and its treatment. A second possibility is that none of the SF-36 subscales recorded specific GH-dependent impairment and treatment changes in subjective QoL in $\mathrm{CO}$ patients, suggesting a lack of responsiveness. The SF-36 is not designed to address issues specific to GHD adults, such as sexual functioning, cognitive functioning, and sleep disorders (31), and therefore seems to have limited sensitivity to changes in QoL in adults with GHD. In this regard, no disease-oriented QoL questionnaire has yet been validated in Japan.

No significant differences between $\mathrm{GH}$ and placebo treatment were seen in changes in any subscale, except for the mental health domain in $\mathrm{CO}$ patients during the double-blind phase. The double-blind study was originally powered to detect between-treatment differences in lean body mass, and was therefore underpowered for assessing the effects of GH on QoL. During the double-blind phase, serum IGF-I levels were elevated above the upper limit of the reference range in eight of the fourteen $\mathrm{AO}$ patients of the $\mathrm{GH}$ group, and nine AO patients experienced oedema and/or painful events such as arthralgia and muscle pain (8). These events may have been caused by the relatively high $\mathrm{GH}$ dose and supraphysiological IGF-I levels due to the fixed dose regimen, and have had a particularly adverse effect on QoL in AO patients. The results of our first doubleblind study therefore appear to be inconclusive with regard to physiological GH replacement. Throughout the study, a negative correlation between bodily pain score change and IGF-I change was seen in CO patients, also indicating the possible impact of IGF-I related adverse events on this domain. At the end of the open study, individualized doses and treatment effects had stabilized, and mean IGF-I levels were within the normal range in both onset groups. For analyses of evaluating responsiveness, we calculated change scores in QoL subscales throughout both phases from all patients, pooled regardless of treatment group in the double-blind phase, yielding greater power to detect changes within each onset group. Consequently, the analyses performed were identical to those for an open-label study without control and the results should be accordingly interpreted with caution for potential bias.

Controversy has existed over whether GHD per se affects QoL in hypopituitary adults and whether GH can reverse it. Several subscale scores were reduced at baseline in our study population, which could be specifically ascribed to GHD but also possibly to confounding factors, including other hormonal deficits and their inadequate replacement, and the underlying causes of hypopituitarism, such as tumors and their invasive treatments. External responsiveness alone gives little information to indicate the effects of treatment (7); nevertheless, changes in a QoL instrument responsive to changes in a valid external 
clinical index would indicate efficacy of the treatment on QoL, providing the changes in the disease condition seen with treatment are directly related to changes in the external parameter. Given that excess fat and visceral adiposity are the major symptoms of adult GHD, are improved by GH replacement, and contribute to poor body image leading to reduced self-estimation, one may postulate a causal relationship between GH replacement and the improvement of poor subjective QoL; this assumption would need to be confirmed in further clinical studies.

In conclusion, we have demonstrated that Japanese hypopituitary adults with GHD displayed onset differences in the degree of QoL impairment at baseline, similar to Caucasian patients, and that the positive changes in physical functioning and general health domains were significantly correlated with fat mass reduction under GH replacement. The effect of fat mass reduction on these subscales was greater in $\mathrm{AO}$ than $\mathrm{CO}$ patients, indicating heterogeneity between onset types in the external responsiveness of the QoL subscales to body composition. Given that excess fat mass is an established clinical index of GHD, these subscales appear to be valid measures of the perceived QoL changes associated with changes in clinical condition in adults with AO GHD. Nonetheless, our observations are based on a limited number of patients and the data must be considered preliminary. Development of a diseasespecific questionnaire for Japanese adults with GHD will enable further research for verification and individualized disease management.

\section{Acknowledgements}

We are grateful to the following study sites and investigators for their participation and thoughtful advice during the conduct of the study: Dr Kenji Fujieda, Asahikawa Medical College, Hokkaido; Dr Kazumichi Onigata, Gunma University, Gunma; Dr Kazue Takano, Tokyo Women's Medical University, Tokyo; Dr Akira Teramoto, Dr Hitoshi Sugihara, and Dr Yukashi Ohki, Nippon Medical School, Tokyo; Dr Yoshikatsu Eto, Tokyo Jikei University School of Medicines, Tokyo; Dr Yasunori Ozawa, Dr Shozo Yamada, and Dr Susumu Yokoya, Toranomon Hospital, Tokyo; Dr Toshiaki Tanaka, National Center for Child Health and Development, Tokyo; Dr Yutaka Oki, Hamamatsu University School of Medicine, Shizuoka; Dr Kiyoshi Hashizume, Shinshu University, Nagano; Dr Akira Shimatsu, National Hospital Organization, Kyoto Medical Center, Kyoto; Dr Soji Kasayama, Dr Sotaro Mushiake, and Dr Tokuzo Harada, Osaka University, Osaka; Dr Genzo Iguchi, and Dr Keiji Iida, Kobe University, Hyogo; Dr Yuzuru Kato, Shimane University, Shimane; Dr Yoshiki Seino, and Dr Hiroyuki Tanaka, Okayama University, Okayama; Dr Hajime Nawata, Kyushu University, Fukuoka; Dr Noritaka Iwatani, Kumamoto University, Kumamoto; Dr Hideki
Katakami, University of Miyazaki Miyazaki Medical College, Miyazaki; Dr Takashi Yoneda, Kanazawa University, Ishikawa; Dr Osamu Arisaka, Dokkyo University School of Medicine, Tochigi; Dr Keinosuke Fujita, Osaka City General Hospital, Osaka; and Dr Hitoshi Kohno, Fukuoka Children's Hospital, Fukuoka. We also gratefully acknowledge the support provided by Andrea Attanasio and Peter Bates for manuscript development, and the dedication and concerted effort of colleagues at Eli Lilly. The studies were conducted by Eli Lilly Japan.

\section{References}

1 Consensus guidelines for the diagnosis and treatment of adults with growth hormone deficiency: summary statement of the Growth Hormone Research Society Workshop on Adult Growth Hormone Deficiency. Journal of Clinical Endocrinology and Metabolism $1998 \mathbf{8 3}$ 379-381.

2 Chrisoulidou A, Kousta E, Beshyah S, Robinson S \& Johnston DG. How much, and by what mechanisms, does growth hormone replacement improve the quality of life in GH-deficient adults? Bailliere's Clinical Endocrinology and Metabolism $1998 \mathbf{1 2}$ 261-279.

3 Hull KL \& Harvey S. Growth hormone therapy and quality of life: possibilities, pitfalls and mechanisms. Journal of Endocrinology $2003179311-333$.

4 Woodhouse LJ, Mukherjee A, Shalet SM \& Ezzat S. The influence of growth hormone status on physical impairments, functional limitations, and health-related quality of life in adults. Endocrine Reviews 200627 287-317.

5 Attanasio AF, Lamberts SWJ, Matranga AMC, Birkett MA, Bates PC, Valk NK, Hilsted J, Bengtsson BA \& Strasburger CJ. Adult growth hormone $(\mathrm{GH})$-deficient patients demonstrate heterogeneity between childhood onset and adult onset before and during human GH treatment. Adult Growth Hormone Deficiency Study Group. Journal of Clinical Endocrinology and Metabolism $1997 \mathbf{8 2} 82-88$.

6 Chihara K, Kato Y, Kohno H, Takano K, Tanaka T, Teramoto A \& Shimatsu A. Efficacy and safety of growth hormone $(\mathrm{GH})$ in the treatment of adult Japanese patients with $\mathrm{GH}$ deficiency: a randomised, placebo-controlled study. Growth Hormone $\mathcal{E} I G F$ Research 200616 132-142.

7 Husted JA, Cook RJ, Farewell VT \& Gladman DD. Methods for assessing responsiveness: a critical review and recommendations. Journal of Clinical Epidemiology 200053 459-468.

8 Chihara K, Koledova E, Shimatsu A, Kato Y, Kohno H, Tanaka T, Teramoto A, Bates PC \& Attanasio AF. Adult GH deficiency in Japanese patients: effects of $\mathrm{GH}$ treatment in a randomised, placebo-controlled trial. European Journal of Endocrinology 2004 151 343-350.

9 Chihara K, Koledova E, Shimatsu A, Kato Y, Kohno H, Tanaka T, Teramoto A, Bates PC \& Attanasio AF. An individualized GH dose regimen for long-term GH treatment in Japanese patients with adult GH deficiency. European Journal of Endocrinology 2005153 $57-65$.

10 Fukuhara S \& Suzukamo Y. Manual of SF-36v2 Japanese version. Kyoto: Institute for Health Outcomes and Process Evaluation Research 2004.

11 Rosilio M, Blum WF, Edwards DJ, Shavrikova EP, Valle D, Lamberts SWJ, Eva Erfurth M, Webb SM, Ross RJ, Chihara K, Henrich G, Herschbach P \& Attanasio AF. Long-term improvement of quality of life during growth hormone $(\mathrm{GH})$ replacement therapy in adults with GH deficiency, as measured by questions on life satisfaction-hypopituitarism (QLS-H). Journal of Clinical Endocrinology and Metabolism 200489 1684-1693. 
12 Dekkers OM, Biermasz NR, Smit JW, Groot LE, Roelfsema F, Romijn JA \& Pereira AM. Quality of life in treated adult craniopharyngioma patients. European Journal of Endocrinology 2006154 483-489.

13 Sandberg DE, MacGillivray MH, Clopper RR, Fung C, LeRoux L \& Alliger DE. Quality of life among formerly treated childhood onset growth hormone-deficient adults: a comparison with unaffected siblings. Journal of Clinical Endocrinology and Metabolism $1998 \mathbf{8 3}$ 1134-1142.

14 Wiren L, Johannsson G \& Bengtsson BA. A prospective investigation of quality of life and psychological well-being after the discontinuation of $\mathrm{GH}$ treatment in adolescent patients who had GH deficiency during childhood. Journal of Clinical Endocrinology and Metabolism 200186 3494-3498.

15 Johannsson G, Albertsson-Wikland K \& Bengtsson BA. Discontinuation of growth hormone (GH) treatment: metabolic effects in GH-deficient and GH-sufficient adolescent patients compared with control subjects. Swedish Study Group for Growth Hormone Treatment in Children. Journal of Clinical Endocrinology and Metabolism 199984 4516-4524.

16 Pedreira CC, Stargatt R, Maroulis H, Rosenfeld J, Maixner W, Warne GL \& Zacharin MR. Health related quality of life and psychological outcome in patients treated for craniopharyngioma in childhood. Journal of Pediatric Endocrinology and Metabolism $20061915-24$.

17 Norman GR, Stratford P \& Regehr G. Methodological problems in the retrospective computation of responsiveness to change: the lesson of Cronbach. Journal of Clinical Epidemiology $1997 \mathbf{5 0}$ 869-879.

18 Ahmad AM, Hopkins MT, Thomas J, Ibrahim H, Fraser WD \& Vora JP. Body composition and quality of life in adults with growth hormone deficiency; effects of low-dose growth hormone replacement. Clinical Endocrinology 200154 709-717.

19 Mukherjee A, Adams JE, Smethurst L \& Shalet SM. Interdependence of lean body mass and total body water, but not quality of life measures, during low dose GH replacement in GH-deficient adults. European Journal of Endocrinology $2005153661-668$.

20 Davies JS, Obuobie K, Smith J, Rees DA, Furlong A, Davies N, Evans LM \& Scanlon MF. A therapeutic trial of growth hormone in hypopituitary adults and its influence upon continued prescription by general practitioners. Clinical Endocrinology 200052 295-303.

21 Murray RD, Skillicorn CJ, Howell SJ, Lissett CA, Rahim A, Smethurst LE \& Shalet SM. Influences on quality of life in GH deficient adults and their effect on response to treatment. Clinical Endocrinology $1999 \mathbf{5 1} 565-573$.
22 Kendall-Taylor P, Jonsson PJ, Abs R, Erfurth EM, KoltowskaHaggstrom M, Price DA \& Verhelst J. The clinical, metabolic and endocrine features and the quality of life in adults with childhoodonset craniopharyngioma compared with adult-onset craniopharyngioma. European Journal of Endocrinology 2005152 557-567.

23 Mukherjee A, Tolhurst-Cleaver S, Ryder WD, Smethurst L \& Shalet SM. The characteristics of quality of life impairment in adult growth hormone $(\mathrm{GH})$-deficient survivors of cancer and their response to GH replacement therapy. Journal of Clinical Endocrinology and Metabolism $2005901542-1549$.

24 Holmes SJ, McKenna SP, Doward LC, Hunt SM \& Shalet SM. Development of a questionnaire to assess the quality of life of adults with growth hormone deficiency. Endocrinology and Metabolism 19952 63-69.

25 Herschbach P, Henrich G, Strasburger CJ, Feldmeier H, Martin F, Attanasio AM \& Blum WF. Development and psychometric properties of a disease-specific quality of life questionnaire for adult patients with growth hormone deficiency. European Journal of Endocrinology 2001145 255-265.

26 Han TS, Tijhuis MAR, Lean MEJ \& Seidell JC. Quality of life in relation to overweight and body fat distribution. American Journal of Public Health $1998 \mathbf{8 8} 1814-1820$.

27 Lebrun CE, van der Schouw YT, de Jong FH, Pols HA, Grobbee DE \& Lamberts SW. Relations between body composition, functional and hormonal parameters and quality of life in healthy postmenopausal women. Maturitas 200655 82-92.

28 Duval K, Marceau P, Perusse L \& Lacasse Y. An overview of obesityspecific quality of life questionnaires. Obesity Reviews 20067 347-360.

29 Lasaite L, Bunevicius R, Lasiene D \& Lasas L. Psychological functioning after growth hormone therapy in adult growth hormone deficient patients: endocrine and body composition correlates. Medicina 200440 740-744.

30 Stouthart PJ, Deijen JB, Roffel M \& Delemarre-van de Waal HA. Quality of life of growth hormone $(\mathrm{GH})$ deficient young adults during discontinuation and restart of GH therapy. Psychoneuroendocrinology 200328 612-626.

31 Ware JE \& Sherbourne CD. The MOS 36-item short-form health survey (SF-36). I. Conceptual framework and item selection. Medical Care 199230 473-483.

Received 12 January 2007

Accepted 29 March 2007 